\title{
Update on non-bismuth quadruple (concomitant) therapy for eradication of Helicobacter pylori
}

This article was published in the following Dove Press journal:

Clinical and Experimental Gastroenterology

13 March 2012

Number of times this article has been viewed

\author{
Javier P Gisbert' \\ Xavier Calvet ${ }^{2}$ \\ 'Department of Gastroenterology, \\ Hospital Universitario de La \\ Princesa, Instituto de Investigación \\ Sanitaria Princesa (IP), and Centro \\ de Investigación Biomédica en \\ Red de Enfermedades Hepáticas y \\ Digestivas (CIBEREHD), Madrid, Spain; \\ ${ }^{2}$ Department of Gastroenterology, \\ Hospital de Sabadell, Departament \\ de Medicina, Universitat Autònoma \\ de Barcelona and Centro de \\ Investigación Biomédica en Red de \\ Enfermedades Hepáticas y Digestivas \\ (CIBEREHD), Barcelona, Spain
}

Background: Traditional standard triple therapy for Helicobacter pylori (H. pylori) infection (proton pump inhibitor-clarithromycin-amoxicillin) can easily be converted to non-bismuth quadruple (concomitant) therapy by the addition of a nitroimidazole twice daily.

Aim: To critically review evidence on the role of non-bismuth quadruple therapy (proton pump inhibitor-clarithromycin-amoxicillin-nitroimidazole) in the treatment of $H$. pylori infection.

Methods: Bibliographical searches were performed in MEDLINE and relevant congresses up to December 2011. We performed a meta-analysis of the studies evaluating the concomitant therapy, and of the randomized controlled trials comparing the concomitant and the standard triple therapy.

Results: A meta-analysis of 19 studies (2070 patients) revealed a mean $H$. pylori cure rate (intention-to-treat) of $88 \%$ (95\% confidence interval from $85 \%$ to $91 \%$ ) for non-bismuth quadruple therapy. We performed a meta-analysis of the randomized controlled studies comparing the concomitant (481 patients) and the standard triple therapy (503 patients). The former was more effective than the latter: $90 \%$ versus $78 \%$ (intention-to-treat analysis). Results were homogeneous $\left(I^{2}=0 \%\right)$. The odds ratio for this comparison was 2.36 ( $95 \%$ confidence interval from 1.67 to 3.34 ). A tendency toward better results with longer treatments (7-10 days versus 3-5 days) has been observed, so it seems reasonable to recommend the length of treatment achieving the highest cure rates (10 days). Clarithromycin resistance may reduce the efficacy of non-bismuth quadruple therapy, although the decrease in eradication rates seems to be far lower than in standard triple therapy. Experience with the non-bismuth quadruple therapy in patients with metronidazole-resistant strains is still very limited.

Conclusion: Non-bismuth quadruple (concomitant) therapy appears to be an effective, safe, and well-tolerated alternative to triple therapy and is less complex than sequential therapy. Therefore, this regimen appears well suited for use in settings where the efficacy of triple therapy is unacceptably low.

Keywords: Helicobacter pylori, concomitant therapy, sequential therapy, clarithromycin, metronidazole, non-bismuth quadruple

\section{Introduction}

Helicobacter pylori $(H$. pylori) infects approximately $50 \%$ of the adult population and is associated with a wide range of upper gastrointestinal diseases including gastritis, peptic ulcer disease, and gastric cancer. ${ }^{1}$ The most widely recommended treatment in international guidelines for the eradication of $H$. pylori is the so called standard, or proton pump inhibitor (PPI)-based, triple therapy, which combines two antibiotics (clarithromycin plus amoxicillin or metronidazole) with a PPI for at least 7 days. ${ }^{2-6}$ However, since the microorganism was discovered, the eradication rates
Correspondence: Javier P Gisbert Playa de Mojácar 29. Urb. Bonanza, 28669 Boadilla del Monte, Madrid, Spain

Tel +34 9|30939| I

$\mathrm{Fax}+34914022299$

Email gisbert@meditex.es 
have fallen considerably with this regimen. ${ }^{7,8}$ Two recent double-blind, US multicenter studies both found disappointingly low eradication rates with standard therapy $(77 \%),{ }^{9,10}$ and two meta-analyses including more than 53,000 patients revealed the cure rate to be below $80 \% .{ }^{11,12}$ Therefore, the ethics of continued use of standard triple therapy have recently been questioned, and alternative approaches have been recommended. ${ }^{13}$ Attempts to increase the duration of triple therapy, thus prolonging exposure to antibiotics, have achieved controversial results, but have not generally resulted in remarkable benefits. ${ }^{14,15}$ Consequently, new strategies to improve first-line treatment are still urgently needed.

One recent innovation, postulated as an alternative to standard triple therapy, is sequential treatment, which involves a simple dual regimen including a PPI plus amoxicillin for the first 5 days followed by a triple regimen including a PPI, clarithromycin, and tinidazole for the following 5 days. ${ }^{16}$ Several randomized clinical trials (including pooled-data analyses and meta-analyses) have demonstrated that a sequential regimen is more effective than standard triple therapy. ${ }^{17-21}$ Therefore, some guidelines have proposed sequential therapy as an alternative to standard triple therapy for the eradication of $H$. pylori. ${ }^{22}$ However, a recent update of previous meta-analyses performed by a Cochrane Collaboration group ${ }^{23}$ found that the results obtained with the sequential regimen were clearly heterogeneous, and that several recently published studies were unable to demonstrate differences between sequential and standard triple therapy. So, although the overall mean eradication rate with the sequential regimen was nearly $90 \%$, a tendency towards lower efficacy with this regimen was observed in the more recent studies. ${ }^{24-27}$

Moreover, a relevant question is whether it is necessary to provide the drugs sequentially or if the four constituent components of sequential therapy can be given concurrently. ${ }^{28,29}$ In other words, does sequential administration represent an advantage or does it make therapy more complicated than necessary? ${ }^{13,30}$ In this regard, the triple combination of clarithromycin plus amoxicillin and a nitroimidazole with a PPI (but without bismuth) has previously been examined as a nonsequential regimen, which proved effective. The concept of a "non-bismuth quadruple regimen" or "concomitant" regimen (the term used hereafter) has recently resurfaced. ${ }^{31}$ Traditional standard triple therapy (PPI-clarithromycinamoxicillin) can easily be converted to concomitant therapy by the addition of $500 \mathrm{mg}$ of metronidazole or tinidazole twice daily. ${ }^{32}$
The aim of the present article is to critically review evidence on the role of concomitant therapy in the treatment of $H$. pylori infection. We review the following aspects: rationale for use, efficacy of the regimen and the variables affecting it, comparison between the concomitant regimen and standard triple and sequential therapy, and finally, limitations of the concomitant regimen.

\section{Search strategy}

Bibliographical searches were performed in MEDLINE up to December 2011 using the following keywords (all fields): ("Helicobacter pylori" OR "H. pylori") AND concomitant OR concurrent OR quadruple OR (clarithromycin AND [amoxicillin OR amoxycillin]) AND (metronidazole OR tinidazole OR nitroimidazole). Articles published in any language were included. Reference lists from the trials selected in the electronic search were hand-searched to identify further relevant trials. We also conducted a manual search of abstracts from the scientific meetings of the International Workshop of the European Helicobacter Study Group, the United European Gastroenterology Week, and the American Digestive Disease Week. Abstracts of the articles selected in each of these multiple searches were reviewed, and those meeting the inclusion criteria were selected. References from reviews on $H$. pylori treatment with the concomitant regimen and from the works selected for the study were also examined to identify articles meeting the inclusion criteria. In the case of duplicate reports or studies obviously reporting results from the same study population, only the latest published data were used.

\section{Rationale/historical perspective of the concomitant regimen}

In 1998, two groups of investigators, one in Germany and the other in Japan, proposed that a PPI, amoxicillin, clarithromycin, and nitroimidazole be given concurrently as a nonsequential four-drug, three-antibiotic, non-bismuth-containing quadruple regimen. ${ }^{33,34}$ Despite the short duration of therapy ( 5 days on average), this approach provided high cure rates ( $>90 \%$ by intention-to-treat).

The efficacy of a triple regimen (PPI, clarithromycin, and a nitroimidazole) was known to be inversely related to bacterial load, and higher eradication rates were achieved in patients with a low bacterial density in the stomach. ${ }^{35-37}$ Therefore, the addition of amoxicillin lowered bacterial load in the stomach, with the consequent improvement in the efficacy of the short course of triple therapy. ${ }^{38}$ In other words, concurrent administration of the three antibiotics as 
concomitant therapy proved more efficacious than when they were administered separately. ${ }^{39}$

Proponents of sequential treatment (amoxicillin for 5 days, followed by clarithromycin plus a nitroimidazole for a further 5 days) argue that initial use of amoxicillin could provide a key advantage in the eradication of $H$. pylori. ${ }^{18}$ namely, prevention of the selection of secondary clarithromycin resistance. ${ }^{40}$ Indeed, it is known that bacteria can develop efflux channels for clarithromycin, which rapidly transfer the drug out of the bacterial cell, preventing the binding of the antibiotic to the ribosome. ${ }^{41-43}$ It has been speculated that the disruption of the cell wall caused by amoxicillin prevents the development of efflux channels by damaging the cell wall of the bacterium. In theory, this disruption could help to improve the efficacy of clarithromycin in the second phase of treatment. ${ }^{40,44}$ However, the improved effect with sequential (and concomitant) therapy - as compared with standard triple therapy - may not be due to sequential administration itself, but to the larger number of antibiotics (three drugs) to which the organism is exposed or to the use of a nitroimidazole, which is not contained in the standard triple-drug regimen. ${ }^{45,46}$

\section{Efficacy of the concomitant regimen for eradication of $\mathrm{H}$. pylori}

Studies evaluating the efficacy of the concomitant regimen are summarized in Table 1..$^{33,34,47-63}$ These studies were performed in different countries in Europe, Asia, and America, and most were randomized controlled trials. Similar concomitant regimens were prescribed, with only minor modifications, namely, the PPI (omeprazole, lansoprazole, rabeprazole, or esomeprazole) and the nitroimidazole (metronidazole or tinidazole). However, duration of treatment varied markedly between 3 and 14 days (see below). Our analysis of the 19 studies (2070 patients) revealed a mean $H$. pylori cure rate (intention-to-treat) of $88 \%$ (95\% confidence interval [95\% $\mathrm{CI}$ ] from $85 \%$ to $91 \%$ ) (Table 2). The data were combined using the generic inverse variance method, which involves a weighted average of the effect estimates from the individual studies. The weight for each study is taken to be the inverse of the variance (one divided by the square of the standard error) of the effect estimate. As results were heterogeneous $\left(P<0.001 ; I^{2}=80 \%\right)$, a random effect model (DerSimonian and Laird) was applied to perform the meta-analysis (using Review Manager 5.0.25, developed by the Cochrane Collaboration).

From those studies, the one performed in Latin America (including patients from Chile, Colombia, Costa
Rica, Honduras, Mexico, and Nicaragua) had markedly disappointing results, with a $74 \%$ eradication rate. ${ }^{57}$ The explanation for this outlier is unclear, as information on the antibiotic susceptibility of $H$. pylori is not available in the article. Furthermore, even though the treatment lasted only 5 days, other studies with the same period of administration performed some years ago obtained excellent results $(>90 \%)$ (see below for a more detailed discussion on the duration of treatment). We might speculate that 5-day concomitant regimens were effective enough a decade ago, but that increased antibiotic resistance rates have revealed the need for longer regimens.

A second outlier study was performed by Toros et $\mathrm{al}^{60}$ in Turkey, where only a $75 \%$ eradication rate was achieved despite the fact that high doses of metronidazole $(500 \mathrm{mg}$ three times daily) were used and a 14-day regimen was prescribed. It should be taken into consideration that, in Turkey, results with the standard triple therapy have also been far lower than expected. Thus, in a large randomized trial performed in Turkey, H. pylori eradication rates achieved with a PPI-clarithromycin-amoxicillin regimen for 7 and 14 days were only $24 \%$ and $43 \%$, respectively. ${ }^{64}$

Finally, a third outlier study was performed by Choi et $\mathrm{al}^{63}$ in Korea, where only a $63 \%$ cure rate was reported, although the study has not yet been published as a peer-reviewed article and only 38 patients were included.

The cure rates recorded in the remaining studies were $>80 \%$ and even $>90 \%$. In fact, if these three outlier studies are excluded, the mean eradication rate (intentionto-treat) of the remaining 16 studies increased to $91 \%$, and the interstudy heterogeneity almost completely disappeared $\left(I^{2}=10 \%\right)$.

\section{Effects of different variables on the efficacy of concomitant therapy}

The efficacy of the concomitant regimen on $H$. pylori eradication depends on several factors.

\section{Clarithromycin resistance}

Resistance rates for antimicrobial agents rise with indiscriminate use, and clarithromycin resistance may be due to the widespread use of this agent for upper respiratory tract infections. ${ }^{65,66}$ Antimicrobial resistance is largely responsible for the poor eradication rates with standard triple therapy. ${ }^{67-69}$ Culture and antimicrobial sensitivity testing of $H$. pylori are not widely available and, when they are, they may not produce any clear clinical benefit..$^{32,70-72}$ One meta-analysis reported an almost $60 \%$ decline in eradication rates with 


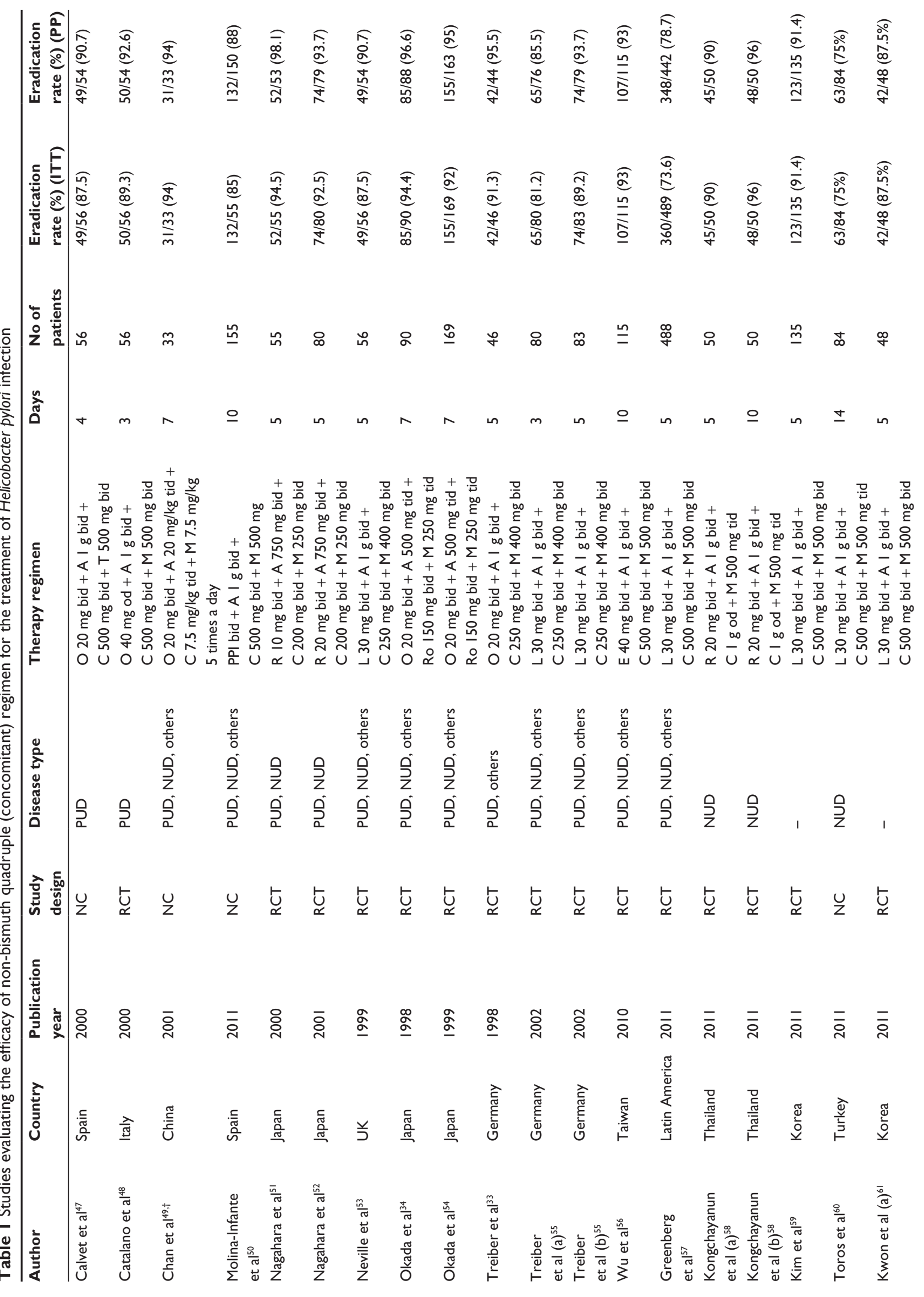




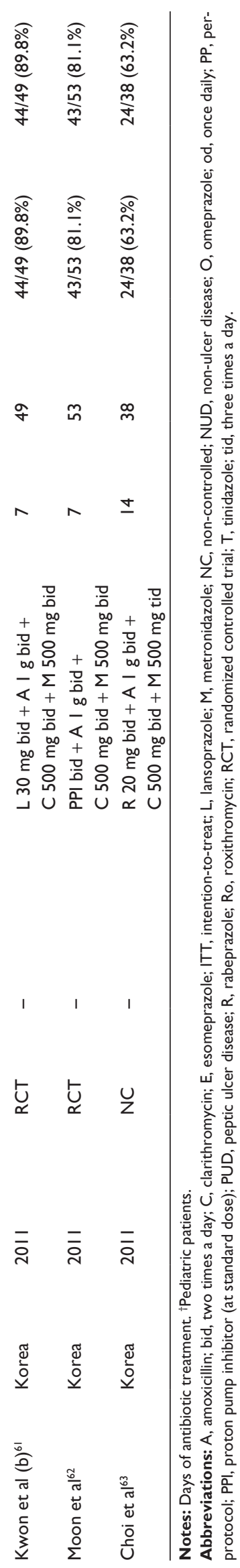

standard triple therapy if clarithromycin resistance was present. ${ }^{67,73}$ Therefore, the use of standard triple therapy has been recommended only in those areas where clarithromycin resistance is lower than $15 \%-20 \%{ }^{2}$

Clarithromycin resistance reduces the efficacy of sequential therapy, although the decrease in eradication rates was far lower than in triple therapy. ${ }^{16,18-21,45,74}$ Therefore, the sequential treatment regimen may be preferable to triple therapy when the prevalence of clarithromycin-resistant H. pylori infection is high, which is the case in many developed countries. ${ }^{45}$

This advantage of sequential therapy over standard triple therapy (ie, higher eradication rates among patients with clarithromycin resistance) also seems to be applicable to concomitant therapy. An initial meta-analysis determined the effect of drug resistance on the efficacy of first-line treatment regimens for $H$. pylori and identified the most effective treatments in the presence of drug resistance; the results showed that resistance to clarithromycin or metronidazole may be overcome by using quadruple therapies, especially those containing both clarithromycin and metronidazole. ${ }^{69}$ The effect of clarithromycin resistance on the efficacy of concomitant regimens was negligible, with $95 \%$ efficacy in the clarithromycin-sensitive arm, and $96 \%$ in the clarithromycin-resistant arm. ${ }^{69}$ Nevertheless, this conclusion was based on only two studies. ${ }^{53,54}$ More recently, Wu et al ${ }^{56}$ found no significant effect of antibiotic resistance on the efficacy of concomitant therapy: H. pylori was eradicated in three out of four $(75 \%)$ clarithromycin-resistant patients.

Finally, Molina-Infante et $\mathrm{al}^{75}$ recently compared quadruple concomitant therapy and standard triple therapy for clarithromycin-susceptible $H$. pylori strains. A statistically nonsignificant tendency $(P=0.2)$ to better results was observed with concomitant therapy compared with standard triple therapy, both by per-protocol (88\% versus $78 \%$ ) and by intention-to-treat ( $88 \%$ versus $73 \%$ ) analysis. Therefore, quadruple concomitant therapy may be more effective than standard triple therapy even for clarithromycin-susceptible H. pylori.

Indirect evidence supporting an advantage of a concomitant regimen over a sequential regimen comes from a recent study. ${ }^{50}$ The authors evaluated the efficacy of empiric concomitant therapy in a geographical area (Spain) where sequential therapy had previously proved inefficient (76\% cure rate in a prior study ${ }^{24}$ ). Eradication rates for the concomitant regimen were $88 \%$ by per-protocol analysis and $85 \%$ by intention-to-treat analysis, and the authors concluded that in settings with high clarithromycin resistance $(>5 \%-20 \%)$ and 
Table 2 Meta-analysis of efficacy (intention-to-treat) of studies evaluating the concomitant regimen for the treatment of Helicobacter pylori infection

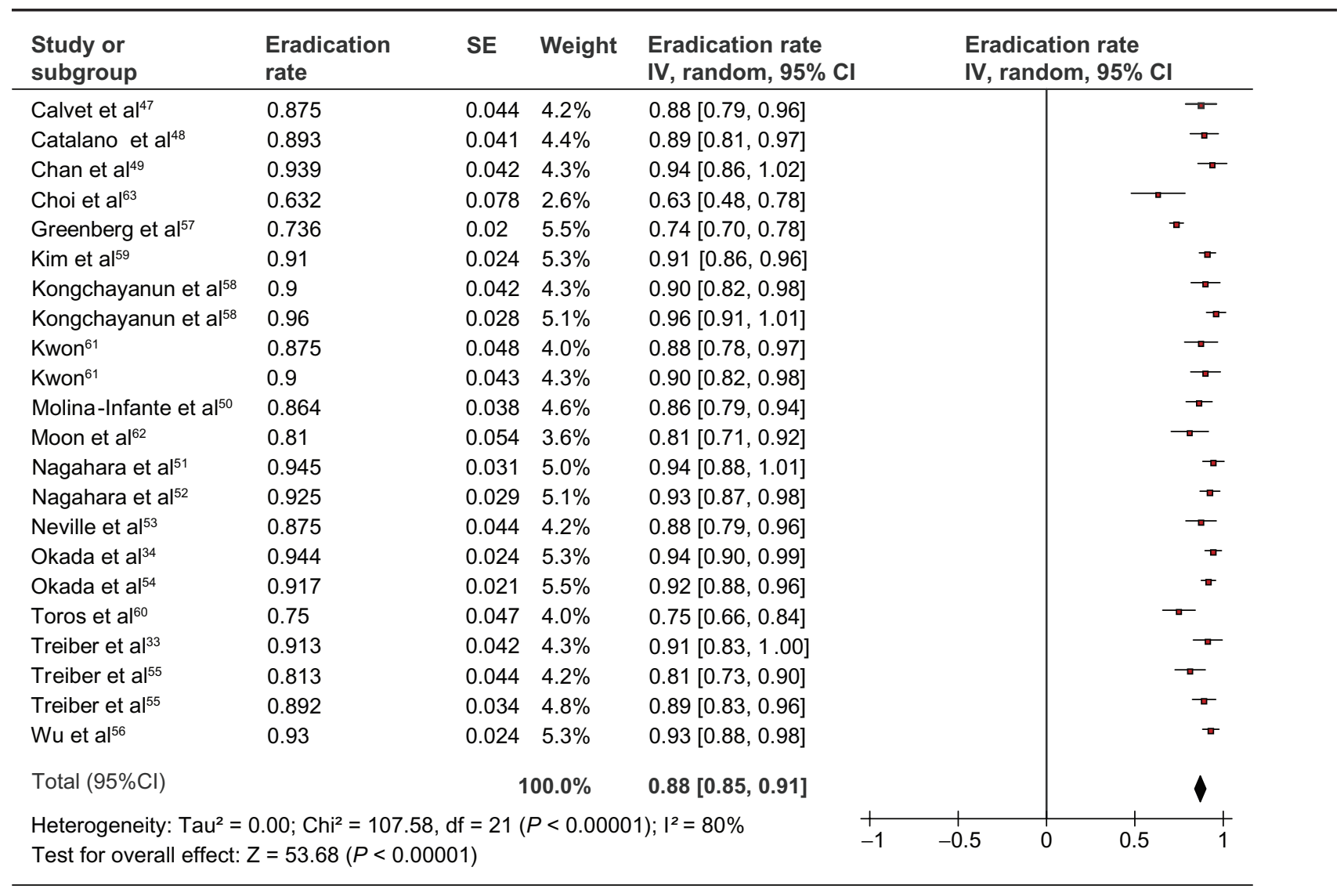

Abbreviations: $\mathrm{Cl}$, confidence interval; IV, inverse variance; SE, standard error.

documented failure of sequential therapy, concomitant therapy may achieve acceptable eradication rates. ${ }^{50}$ The reason for this theoretical advantage of concomitant therapy over sequential therapy (which should be confirmed in randomized controlled trials including both regimens in the same study) may be a lower effect of antibiotic resistance on the eradication rate with concomitant therapy (when all three antibiotics are administered concurrently) or the longer period of time each antibiotic is prescribed ( 5 days in the sequential regimen and $7-10$ days in the 7 to 10 -day concomitant regimen).

\section{Nitroimidazole resistance}

Despite the inclusion of tinidazole, it has been suggested that the sequential regimen may achieve a significantly higher eradication rate than the tinidazole-free standard triple therapies. ${ }^{20}$ On the other hand, experience with concomitant therapy in patients with metronidazoleresistant strains is still very limited. In the study by Neville et al, ${ }^{53}$ similar eradication rates against both metronidazole-sensitive (95\%) and metronidazole-resistant $(85 \%)$ strains were achieved with the concomitant regimen.
Okada et al ${ }^{54}$ found that $H$. pylori was eradicated in 25 out of $27(93 \%)$ patients with metronidazole-resistant strains compared with 130 out of 136 (96\%) patients with metronidazole-sensitive strains. However, Treiber et $\mathrm{al}^{55}$ observed that 5-day concomitant treatment eradicated H. pylori in $90 \%$ of metronidazole-susceptible patients but in only $50 \%(8 / 16)$ of metronidazole-resistant patients. Finally, bismuth-based quadruple therapy has been proposed as a means of overcoming imidazole resistance, and it remains to be seen how concomitant therapy would perform in comparison. ${ }^{76}$

\section{Dual clarithromycin and metronidazole resistance}

Sequential therapy has been reported to be absolutely ineffective in patients with dual resistance (clarithromycin and imidazole).$^{45}$ Primary dual resistance for clarithromycin and imidazole has been shown to produce an eradication rate of $50 \%(2 / 4)$ following 5 days of concomitant therapy ${ }^{55}$ and $75 \%$ (3/4) after 7 days of concomitant therapy. ${ }^{54}$

Comparative studies where both sequential and concomitant regimens are administered are clearly necessary. 
In this respect, Wu et $\mathrm{al}^{56}$ compared the efficacy of sequential and concomitant therapy and analyzed the effects of antibiotic resistance. Dual resistance did not influence the level of eradication in the concomitant therapy group, but significantly affected that of the sequential therapy group. In particular, patients with dual resistance had a significantly lower eradication rate after sequential therapy (present versus absent: $33.3 \%$ versus $95.1 \% ; P<0.0001$ ), but not after concomitant therapy $(75.0 \%$ versus $92.4 \%$, respectively; $P=0.22$; although the low number of patients makes the possibility of a type II error likely).

Finally, Molina-Infante et $\mathrm{al}^{75}$ recently compared quadruple concomitant and sequential therapies for clarithromycinresistant and dual clarithromycin and metronidazole resistant strains. Per-protocol and intention-to-treat eradication rates for clarithromycin-resistant strains with concomitant and sequential treatments were $100 \%(4 / 4)$ and $80 \%(4 / 5)$, and for dual clarithromycin- and metronidazole-resistant strains they were $66 \%(2 / 3)$ and $75 \%(3 / 4)$. Therefore, the authors concluded that both quadruple concomitant and sequential regimens may maintain acceptable eradication rates for clarithromycin-resistant and for dual clarithromycin- and metronidazole-resistant strains.

In summary, concomitant therapy may be more suitable than sequential therapy for patients with dual resistance to antibiotics. Nevertheless, one would suspect that neither concomitant nor sequential therapy would be a good choice in the face of known dual resistance. ${ }^{13}$ In any case, these considerations are based on results from small samples; therefore, more data are needed before a reliable conclusion can be drawn.

\section{Duration of treatment}

Non-bismuth quadruple (concomitant) therapy was originally developed in an attempt to decrease the duration of treatment for $H$. pylori infection. In studies performed in the late 1990s, data from Europe and Japan suggested that a short course of 3-5 days with three antibiotics and a PPI could achieve reasonable eradication rates. ${ }^{66}$

In their meta-analysis (nine studies), Essa et $\mathrm{al}^{77}$ showed that, despite the very short treatment durations of some of the trials, concomitant therapy yielded excellent results but duration of therapy became a significant variable, with longer duration tending to produce higher eradication rates.

The results of the studies included in Tables 1 and 2 show that, depending on the duration of treatment, mean H. pylori eradication rates for concomitant treatment were: 3 days (85\%), 4 days (88\%), 5 days (83\%), 7 days (91\%), and 10 days (90\%). Therefore, a trend toward better results with longer treatments was observed.

The only randomized trial to date that has compared a 5-day regimen of concomitant therapy with a 10-day regimen ${ }^{58}$ revealed a nonsignificant trend for higher cure rates with the longer regimen (96\% with 10 days versus $90 \%$ with 5 days). Although the authors conclude that both durations were "similar", a type II error may not be ruled out, and this $6 \%$ difference may be clinically relevant.

The real benefit of a highly effective first-line therapy is much greater than the raw percentage data suggest. ${ }^{78}$ As safety is similar and the increase in costs relatively low, it seems reasonable to recommend the length of treatment achieving maximal cure rates (10 days), even though the expected improvements will be moderate.

\section{Comparison between the concomitant regimen and the standard triple regimen Efficacy}

Several randomized studies have confirmed the superiority of concomitant therapy over standard triple therapy. A recent meta-analysis ${ }^{77}$ examined nine prospective trials treating H. pylori for up to 7 days with a concomitant regimen (PPImacrolide-imidazole-amoxicillin). Treatment generally lasted 5 days ( 4 days in one study and 7 days in another). Overall, concomitant therapy was effective in $90 \%$ of patients in the intention-to-treat analysis and 93\% in the per-protocol analysis. Pooled estimates of the five randomized controlled trials showed the superiority of concomitant therapy over triple therapy (odds ratio of 2.86; 95\% CI, 1.73-4.73).

We recently updated these analyses with a more recent study $^{59}$ and have performed a meta-analysis including the randomized controlled studies that, to date (December 2011), have compared these two regimens. As summarized in Table 3, 481 patients received the concomitant regimen and 503 the standard triple regimen. The former was more effective than the latter: $90 \%$ versus $78 \%$ in the intention-to-treat analysis. As the results were very homogeneous $\left(I^{2}=0 \%\right)$, a fixed effect model (Peto method) was used to perform the meta-analysis (Review Manager 5.0.25). The odds ratio for this comparison was $2.36(95 \%$ CI, 1.67-3.34) (Table 3).

\section{Tolerance}

In the meta-analysis by Essa et al, ${ }^{77}$ no severe side effects were reported in any of the studies, apart from anaphylactic reactions to medication. ${ }^{34,54,55}$ Mild to moderate side effects were reported in $27 \%-51 \%$ of patients treated with the 
Table 3 Meta-analysis comparing the efficacy (intention-to-treat) of the concomitant regimen with that of standard triple therapy for the eradication of Helicobacter pylori infection

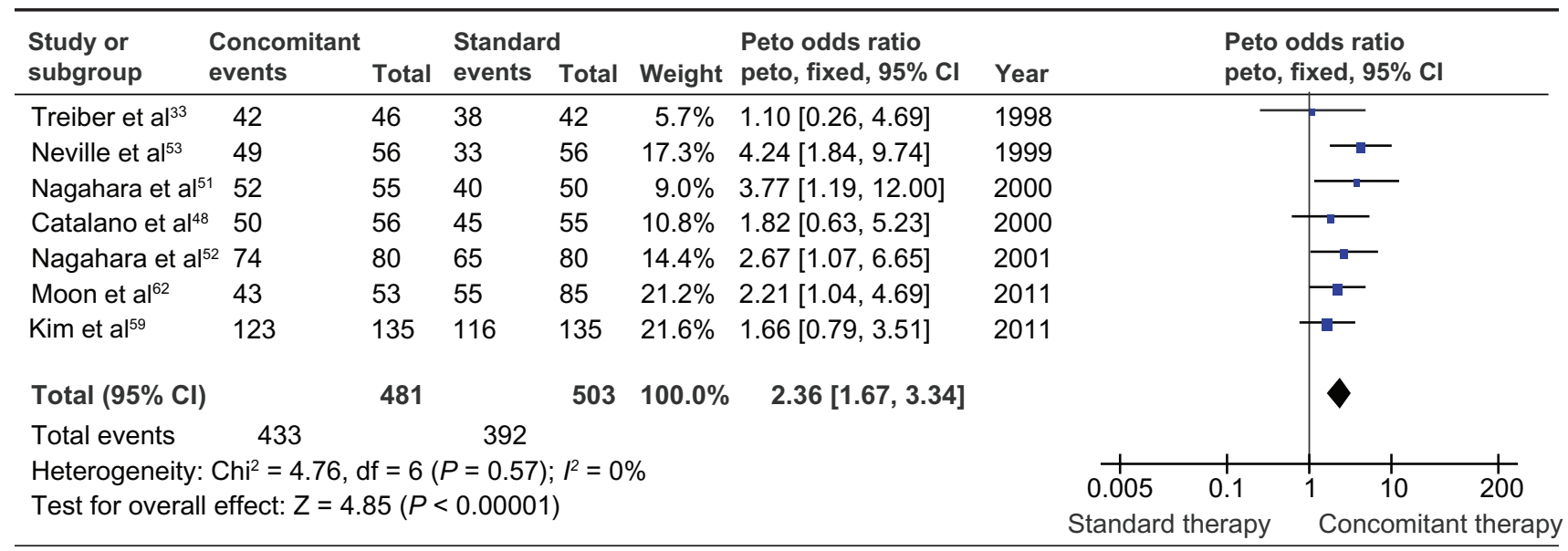

Abbreviation: $\mathrm{Cl}$, confidence interval.

concomitant regimen (compared with $21 \%-48 \%$ of patients treated with triple therapy). ${ }^{77}$ These observations suggest that concomitant and standard triple therapies have a similar safety profile.

\section{Comparison between concomitant and sequential regimens}

One potential problem with sequential therapy is its complexity, as it requires switching from a dual to a triple therapy half way through treatment. Therefore, trials comparing sequential with concomitant therapy using the same combination of drugs are necessary. Such comparisons would address whether the sequential element of sequential quadruple therapy is actually helpful. ${ }^{30} \mathrm{~A}$ direct head-to-head comparison between sequential and concomitant therapy would also tell us which of these two competitors can eventually replace the current first-line triple therapies. ${ }^{79}$

In this respect, Wu et $\mathrm{al}^{56}$ recently performed a multicenter randomized comparison of 10-day sequential therapy with 10-day concomitant therapy, including $232 \mathrm{H}$. pylori-infected patients from three hospitals in Taiwan. Intention-to-treat eradication rates were similar for both regimens: $92 \%$ versus $93 \%$, respectively. Per-protocol cure rates were exactly the same: $93 \%$ with both regimens. The frequency of adverse events was also similar (31\% versus $27 \%)$, as was adherence to therapy ( $96 \%$ versus 98\%). Therefore, the authors concluded that sequential and concomitant administration of the same drugs provides similar results in terms of efficacy and safety and that the sequential administration protocol may produce unnecessary complexity for both patients and physicians compared with concurrent prescription of all the medications from the outset. ${ }^{56}$ The study, however, was performed in a population with a very low rate of clarithromycin and dual clarithromycin-metronidazole resistance; therefore, the potential advantage of concomitant therapy in multiresistant strains may not been adequately appreciated. In fact, the rate of antibiotic resistance in Taiwan is very low, and excellent cure rates (almost 90\%) have also been recently reported with standard triple therapy. ${ }^{80}$

A second randomized study has compared the concomitant regimen (5 days) and the sequential regimen (10 days) in seven Latin American populations ${ }^{57}$ and has reported disappointing results with both regimens (74\% and $76 \%$ cure rates, respectively). By contrast, the eradication rate achieved with the standard triple therapy administered for 14 days was statistically higher (82\%).

\section{Limitations of concomitant therapy}

The results of the aforementioned studies are encouraging, although a number of limitations may affect the strength of their conclusions (see below).

\section{Old data}

Many of the previously mentioned data (see Table 1) are from a decade ago, when the rates of clarithromycin and metronidazole resistance were quite low. ${ }^{66}$ Considering changes in resistance rates, these data may not be valid today. ${ }^{72}$ As few recent data are available from Western populations with current rates of resistance, well-controlled studies are necessary. ${ }^{66}$ Nonetheless, a recently study published in complete journal format reported excellent results: 93\% eradication both by intention-to-treat and by per-protocol analysis with 10 -day concomitant treatment, ${ }^{56}$ suggesting that, at least with the 10-day regimen, favorable results 
may still be obtained. Obviously, further robust assessment across a much broader range of patients is required before concomitant therapy can be generally recommended in clinical practice. ${ }^{16}$

\section{Small sample size and low quality of studies}

The sample in most studies evaluating the concomitant regimen comprises fewer than 100 patients (Table 1). In particular, all the individual studies included in the only meta-analysis published to date had a small sample size. ${ }^{77}$ Furthermore, the quality of the studies is low in most cases. Thus, there are no double-blind randomized controlled trials with this regimen, and only two of the trials included in the meta-analysis by Essa et al were single-blinded, thus limiting the quality of the available evidence. ${ }^{77}$

\section{Insufficient information on the effects of antibiotic resistance}

As most of the published studies failed to evaluate clarithromycin and nitroimidazole resistance, available information is insufficient to truly judge this antimicrobial regimen according to its applicability in populations with high or low antimicrobial resistance.

\section{Limitation of future treatment options after failure of eradication}

All regimens require an adequate back-up or rescue therapy. ${ }^{70,71,79}$ However, it remains unclear how failure of concomitant therapy should be managed. One potential disadvantage of concomitant therapy is that patients with failed eradication would have limited options for further treatment, because they would already have received three different antibiotics: amoxicillin, clarithromycin, and nitroimidazole. ${ }^{17}$ In this respect, it has been suggested that the first choice for eradication treatment should probably not be a regimen combining clarithromycin and metronidazole. ${ }^{70,79}$ Although this regimen is very effective, patients who are not cured will have at least single, and usually double, resistance, ${ }^{81}$ and few logical empirical treatment options are subsequently available. ${ }^{79}$ Some authors have demonstrated that initial regimens containing both clarithromycin and nitroimidazole are associated with significantly worse results overall, with lower eradication rates after logically chosen second-line therapy and sensitivity-directed third-line therapy; the poor results were due to the emergence of multiple resistant strains, as evidenced by culture testing after the second failed course. ${ }^{82}$
However, the recent appearance of levofloxacin may overcome this problem, as levofloxacin-containing rescue therapy constitutes an encouraging empirical second-line or even third-line strategy after multiple previous $H$. pylori eradication failures with key antibiotics such as amoxicillin, clarithromycin, metronidazole, and tetracycline. ${ }^{83-85}$ Zullo et $\mathrm{al}^{86}$ recently performed a pilot study on patients who failed sequential therapy (a regimen including the same antibiotics as concomitant therapy). Following 10-day triple therapy with a PPI, levofloxacin, and amoxicillin, H. pylori infection was successfully cured in $86 \%$ of cases. In another study, Perna et $\mathrm{al}^{87}$ prescribed a 10 -day triple regimen with a PPI, levofloxacin, and amoxicillin in patients in whom first treatment with either standard 10-day triple or sequential therapy (only 10 patients) had failed. H. pylori was eradicated in $73 \%$ of cases, although the authors do not provide separate efficacy rates depending on the first (failure) treatment. Finally, Gisbert et $\mathrm{al}^{88}$ evaluated the efficacy of a second-line levofloxacin-containing triple regimen (PPIamoxicillin-levofloxacin) in 35 patients after "sequential" or "concomitant" treatment failure; $H$. pylori eradication rate was $80 \%$. Respective cure rates for "sequential" and "concomitant" failure regimens were $67 \%$ and $90 \%$.

These data seem to indicate that a triple regimen (PPIlevofloxacin-amoxicillin) is a suitable approach for secondline treatment in patients whose sequential - and probably also concomitant - therapy fails. ${ }^{18,89,90}$ Therefore, the concomitant regimen plus levofloxacin-containing triple therapy may be an adequate therapeutic strategy for the management of $H$. pylori in clinical practice. However, given the rise in resistance to this antibiotic, the prevalence in each country must be taken into account.

Finally, bismuth-based quadruple therapy (ie, PPI, bismuth, tetracycline, and nitroimidazole) could be an alternative in patients whose concomitant therapy fails. Thus, the results of a recent study showed that all patients who had failed sequential therapy (ie, a regimen including the same antibiotics as the concomitant therapy) were able to eradicate the bacterium with bismuth-based quadruple therapy. ${ }^{91}$

\section{Conclusion}

Standard triple therapy is still the most widely used treatment in clinical practice. However, the prevalence of clarithromycin and metronidazole resistance has increased substantially in recent years, and there has been a corresponding decrease in the eradication rate for $H$. pylori infection. Eradication rates are at their lowest levels since a decade ago and are likely to fall further as antimicrobial resistance becomes more 
prevalent worldwide. ${ }^{66}$ It is clear that alternative treatment regimens are urgently needed, particularly for patients with clarithromycin-resistant strains of $H$. pylori $^{92}$

Sequential therapy has been proposed as an alternative to standard triple therapy for eradication of $H$. pylori. However, the sequential approach, which may be more complicated than necessary, does not appear to offer specific advantages. In fact, the first randomized comparison of the sequential and the non-bismuth quadruple concomitant regimens recently concluded that sequential and concomitant administration of the same drugs provide similar results in terms of efficacy and safety.

Several randomized controlled trials (and one metaanalysis) have demonstrated that concomitant therapy is more effective than, and equally well tolerated as, standard triple therapy. Our meta-analysis of 19 studies revealed a mean $H$. pylori cure rate of roughly $90 \%$ for concomitant therapy. A tendency toward better results with longer treatments (7-10 days versus 3-5 days) with the concomitant regimen has been observed, so it seems reasonable to recommend the length of treatment achieving the highest cure rates (10 days).

Clarithromycin resistance may reduce the efficacy of concomitant therapy, although the decrease in eradication rates seems to be far lower than in standard triple therapy. Therefore, it has been suggested that the concomitant regimen may be preferable when the prevalence of clarithromycinresistant $H$. pylori infection is high, which is the case in many developed countries. Experience with the concomitant therapy in patients with metronidazole-resistant strains is still very limited.

Although the aforementioned results are encouraging, a number of limitations should be taken into account: (1) much of the data previously mentioned are relatively old; (2) the number of patients included in most studies evaluating the concomitant regimen is low; (3) the concomitant regimen has not been sufficiently validated in clinical practice; and (4) there is still insufficient information on the effect of antibiotic resistance on efficacy.

In summary, non-bismuth quadruple concomitant therapy appears to be an effective, safe, and well-tolerated alternative to standard triple therapy and is less complex than sequential therapy. Therefore, this regimen appears well suited for use in settings where the efficacy of triple therapy is unacceptably low.

\section{Acknowledgment}

CIBEREHD is funded by the Instituto de Salud Carlos III.

\section{Disclosure}

The authors report no conflicts of interest in this work.

\section{References}

1. McColl KE. Clinical practice. Helicobacter pylori infection. $N$ Engl J Med. 2010;362(17):1597-1604.

2. Malfertheiner P, Megraud F, O'Morain C, et al. Current concepts in the management of Helicobacter pylori infection: the Maastricht III Consensus Report. Gut. 2007;56(6):772-781.

3. Chey WD, Wong BC. American College of Gastroenterology guideline on the management of Helicobacter pylori infection. Am J Gastroenterol. 2007;102(8):1808-1825.

4. Lam SK, Talley NJ. Report of the 1997 Asia Pacific Consensus Conference on the management of Helicobacter pylori infection. J Gastroenterol Hepatol. 1998;13(1):1-12.

5. Coelho LG, Leon-Barua R, Quigley EM. Latin-American Consensus Conference on Helicobacter pylori infection. Latin-American National Gastroenterological Societies affiliated with the Inter-American Association of Gastroenterology (AIGE). Am J Gastroenterol. 2000;95(10):2688-2691.

6. Gisbert JP, Calvet X, Gomollon F, Mones J. Eradication treatment of Helicobacter pylori. Recommendations of the II Spanish Consensus Conference. Med Clin (Barc). 2005;125(8):301-316.

7. Graham DY, Lu H, Yamaoka Y. A report card to grade Helicobacter pylori therapy. Helicobacter. 2007;12(4):275-278.

8. Sasaki M, Ogasawara N, Utsumi K, et al. Changes in 12-year first-line eradication rate of Helicobacter pylori based on triple therapy with proton pump inhibitor, amoxicillin and clarithromycin. J Clin Biochem Nutr. 2010;47(1):53-58.

9. Laine L, Fennerty MB, Osato M, et al. Esomeprazole-based Helicobacter pylori eradication therapy and the effect of antibiotic resistance: results of three US multicenter, double-blind trials. Am J Gastroenterol. 2000;95(12):3393-3398.

10. Vakil N, Lanza F, Schwartz H, Barth J. Seven-day therapy for Helicobacter pylori in the United States. Aliment Pharmacol Ther. 2004;20(1):99-107.

11. Janssen MJ, Van Oijen AH, Verbeek AL, Jansen JB, De Boer WA. A systematic comparison of triple therapies for treatment of Helicobacter pylori infection with proton pump inhibitor/ranitidine bismuth citrate plus clarithromycin and either amoxicillin or a nitroimidazole. Aliment Pharmacol Ther. 2001;15(5):613-624.

12. Laheij RJ, Rossum LG, Jansen JB, Straatman H, Verbeek AL. Evaluation of treatment regimens to cure Helicobacter pylori infection - a metaanalysis. Aliment Pharmacol Ther. 1999;13(7):857-864.

13. Graham DY, Lu H, Yamaoka Y. Therapy for Helicobacter pylori infection can be improved: sequential therapy and beyond. Drugs. 2008; 68(6):725-736.

14. Calvet X, Garcia N, Lopez T, Gisbert JP, Gene E, Roque M. A metaanalysis of short versus long therapy with a proton pump inhibitor, clarithromycin and either metronidazole or amoxycillin for treating Helicobacter pylori infection. Aliment Pharmacol Ther. 2000;14(5): 603-609.

15. Fuccio L, Minardi ME, Zagari RM, Grilli D, Magrini N, Bazzoli F. Meta-analysis: duration of first-line proton-pump inhibitor based triple therapy for Helicobacter pylori eradication. Ann Intern Med. 2007;147(8): 553-562.

16. Gisbert JP, Calvet X, O'Connor A, Megraud F, O'Morain CA. Sequential therapy for Helicobacter pylori eradication: a critical review. J Clin Gastroenterol. 2010;44(5):313-325.

17. Moayyedi P. Sequential regimens for Helicobacter pylori eradication. Lancet. 2007;370(9592):1010-1012.

18. Zullo A, De Francesco V, Hassan C, Morini S, Vaira D. The sequential therapy regimen for Helicobacter pylori eradication: a pooled-data analysis. Gut. 2007;56(10):1353-1357. 
19. Jafri NS, Hornung CA, Howden CW. Meta-analysis: sequential therapy appears superior to standard therapy for Helicobacter pylori infection in patients naive to treatment. Ann Intern Med. 2008;148(12):923-931.

20. Tong JL, Ran ZH, Shen J, Xiao SD. Sequential therapy vs standard triple therapies for Helicobacter pylori infection: a meta-analysis. J Clin Pharm Ther. 2009;34(1):41-53.

21. Gatta L, Vakil N, Leandro G, Di Mario F, Vaira D. Sequential therapy or triple therapy for Helicobacter pylori infection: systematic review and meta-analysis of randomized controlled trials in adults and children Am J Gastroenterol. 2009;104(12):3069-3079; quiz 1080.

22. Caselli M, Zullo A, Maconi G, et al. "Cervia II Working Group Report 2006": guidelines on diagnosis and treatment of Helicobacter pylori infection in Italy. Dig Liver Dis. 2007;39(8):782-789.

23. Gisbert JP, Nyssen OP, McNicholl A, et al. Meta-analysis of sequential vs standard triple therapy for Helicobacter pylori eradication. Helicobacter. 2011;16(Suppl 1):131.

24. Molina-Infante J, Perez-Gallardo B, Fernandez-Bermejo M, et al. Clinical trial: clarithromycin vs levofloxacin in first-line triple and sequential regimens for Helicobacter pylori eradication. Aliment Pharmacol Ther. 2010;31(10):1077-1084.

25. Gao XZ, Qiao XL, Song WC, Wang XF, Liu F. Standard triple, bismuth pectin quadruple and sequential therapies for Helicobacter pylori eradication. World J Gastroenterol. 2010;16(34):4357-4362.

26. Paoluzi OA, Visconti E, Andrei F, et al. Ten and eight-day sequential therapy in comparison to standard triple therapy for eradicating Helicobacter pylori infection: a randomized controlled study on efficacy and tolerability. J Clin Gastroenterol. 2010;44(4):261-266.

27. Romano M, Cuomo A, Gravina AG, et al. Empirical levofloxacincontaining versus clarithromycin-containing sequential therapy for Helicobacter pylori eradication: a randomised trial. Gut. 2010;59(11): 1465-1470.

28. Gisbert JP. Sequential or concomitant therapy for Helicobacter pylori eradication? J Clin Gastroenterol. 2010;44:658-659.

29. Vakil N. Helicobacter pylori treatment: is sequential or quadruple therapy the answer? Rev Gastroenterol Disord. 2008;8(2):77-82.

30. Graham DY, Lu H. Is there a role for sequential in sequential anti-H. pylori therapy? Gastroenterology. 2006;130(6):1930-1931; author reply 1931.

31. Gisbert JP, Calvet X. Review article: non-bismuth quadruple (concomitant) therapy for eradication of Helicobater pylori. Aliment Pharmacol Ther. 2011;34(6):604-617.

32. Graham DY, Shiotani A. New concepts of resistance in the treatment of Helicobacter pylori infections. Nat Clin Pract Gastroenterol Hepatol. 2008;5(6):321-331.

33. Treiber G, Ammon S, Schneider E, Klotz U. Amoxicillin/metronidazole/ omeprazole/clarithromycin: a new, short quadruple therapy for Helicobacter pylori eradication. Helicobacter. 1998;3(1):54-58.

34. Okada M, Oki K, Shirotani T, et al. A new quadruple therapy for the eradication of Helicobacter pylori. Effect of pretreatment with omeprazole on the cure rate. J Gastroenterol. 1998;33(5):640-645.

35. Perri F, Clemente R, Festa V, et al. Relationship between the results of pre-treatment urea breath test and efficacy of eradication of Helicobacter pylori infection. Ital J Gastroenterol Hepatol. 1998;30(2):146-150.

36. Maconi G, Parente F, Russo A, Vago L, Imbesi V, Bianchi Porro G. Do some patients with Helicobacter pylori infection benefit from an extension to 2 weeks of a proton pump inhibitor-based triple eradication therapy? Am J Gastroenterol. 2001;96(2):359-366.

37. Lai YC, Yang JC, Huang SH. Pre-treatment urea breath test results predict the efficacy of Helicobacter pylori eradication therapy in patients with active duodenal ulcers. World J Gastroenterol. 2004;10(7):991-994.

38. Moshkowitz M, Konikoff FM, Peled Y, et al. High Helicobacter pylori numbers are associated with low eradication rate after triple therapy. Gut. 1995;36(6):845-847.

39. Luther J, Chey WD. Sequential therapy for Helicobacter pylori: a two for one deal that's worth the extra effort? Gastroenterology. 2009;136(2): $720-722$.
40. Murakami K, Fujioka T, Okimoto T, Sato R, Kodama M, Nasu M. Drug combinations with amoxycillin reduce selection of clarithromycin resistance during Helicobacter pylori eradication therapy. Int J Antimicrob Agents. 2002;19(1):67-70.

41. Paulsen IT, Brown MH, Skurray RA. Proton-dependent multidrug efflux systems. Microbiol Rev. 1996;60(4):575-608.

42. Webber MA, Piddock LJ. The importance of efflux pumps in bacterial antibiotic resistance. J Antimicrob Chemother. 2003;51(1):9-11.

43. Roberts MC. Resistance to macrolide, lincosamide, streptogramin, ketolide, and oxazolidinone antibiotics. Mol Biotechnol. 2004;28(1) 47-62.

44. De Francesco V, Margiotta M, Zullo A, et al. Clarithromycin-resistant genotypes and eradication of Helicobacter pylori. Ann Intern Med. 2006;144(2):94-100.

45. Vaira D, Zullo A, Vakil N, et al. Sequential therapy versus standard triple-drug therapy for Helicobacter pylori eradication: a randomized trial. Ann Intern Med. 2007;146(8):556-563.

46. Summaries for patients. Sequential therapy versus standard tripledrug therapy for Helicobacter pylori eradication. Ann Intern Med. 2007; 146(8):I20.

47. Calvet X, Tito L, Comet R, Garcia N, Campo R, Brullet E. Four-day, twice daily, quadruple therapy with amoxicillin, clarithromycin, tinidazole and omeprazole to cure Helicobacter pylori infection: a pilot study. Helicobacter. 2000;5(1):52-56.

48. Catalano F, Branciforte G, Catanzaro R, Cipolla R, Bentivegna C, Brogna A. Helicobacter pylori-positive duodenal ulcer: three-day antibiotic eradication regimen. Aliment Pharmacol Ther. 2000;14(10): 1329-1334.

49. Chan KL, Zhou H, Ng DK, Tam PK. A prospective study of a oneweek non-bismuth quadruple therapy for childhood Helicobacter pylori infection. J Pediatr Surg. 2001;36(7):1008-1011.

50. Molina-Infante J, Pazos-Pacheco C, Perez-Gallardo B, et al. Efficacy of 10-day non-bismuth quadruple "concomitant" regimen as first-line therapy for Helicobacter pylori infection in a setting with high rates of clarithromycin and dual clarithromycin and metronidazole resistance. Helicobacter. 2011;16(Suppl 1):133.

51. Nagahara A, Miwa H, Ogawa K, et al. Addition of metronidazole to rabeprazole-amoxicillin-clarithromycin regimen for Helicobacter pylori infection provides an excellent cure rate with five-day therapy. Helicobacter. 2000;5(2):88-93.

52. Nagahara A, Miwa H, Yamada T, Kurosawa A, Ohkura R, Sato N. Five-day proton pump inhibitor-based quadruple therapy regimen is more effective than 7-day triple therapy regimen for Helicobacter pylori infection. Aliment Pharmacol Ther. 2001;15(3):417-421.

53. Neville PM, Everett S, Langworthy H, et al. The optimal antibiotic combination in a 5-day Helicobacter pylori eradication regimen. Aliment Pharmacol Ther. 1999;13(4):497-501.

54. Okada M, Nishimura H, Kawashima M, et al. A new quadruple therapy for Helicobacter pylori: influence of resistant strains on treatment outcome. Aliment Pharmacol Ther. 1999;13(6):769-774.

55. Treiber G, Wittig J, Ammon S, Walker S, van Doorn LJ, Klotz U. Clinical outcome and influencing factors of a new short-term quadruple therapy for Helicobacter pylori eradication: a randomized controlled trial (MACLOR study). Arch Intern Med. 2002;162(2): 153-160.

56. Wu DC, Hsu PI, Wu JY, et al. Sequential and concomitant therapy with 4 drugs are equally effective for eradication of $\mathrm{H}$. pylori infection. Clin Gastroenterol Hepatol. 2010;8:36-41.

57. Greenberg ER, Anderson GL, Morgan DR, et al. 14-day triple, 5-day concomitant, and 10-day sequential therapies for Helicobacter pylori infection in seven Latin American sites: a randomised trial. Lancet. 2011;378(9790):507-514.

58. Kongchayanun C, Mahachai V, Pornthisarn B, Amornsawadwattana S, Vilaichone RK. Efficacy of 10-day and 5-day concomitant therapy for Helicobacter pylori eradication in Thai patients with non ulcer dyspepsia. Gastroenterology. 2011;140(Suppl 1):879. 
59. Kim SY, Lee SW, Kwon BS, et al. Comparative study of Helicobacter pylori eradication rates of 5-day quadruple "Concomitant" therapy and 7-day standard triple therapy. Gastroenterology. 2011; 140(Suppl 1):878.

60. Toros AB, Ince AT, Kesici B, Saglam M, Polat Z, Uygun A. A new modified concomitant therapy for Helicobacter pylori eradication in Turkey. Helicobacter. 2011;16(3):225-228.

61. Kwon BS, Park EB, Lee DH, et al. Effectiveness of 5-day and 7-day quadruple "concomitant" therapy regimen for Helicobacter pylori infection in Korea. Helicobacter. 2011;16(Suppl 1):135.

62. Moon B, Lim H, Lee S, Han K, Chung J, Lee Y. Efficacy of concomitant nonbithmuth-based quadruple therapy as first-line treatment for eradication of Helicobacter pylori. Helicobacter. 2011;16(Suppl 1):131.

63. Choi C, Lee D, Chon I, et al. Concomitant therapy was more effective than ppi-base triple therapy in Korea: a preliminary report. Helicobacter. 2011;16(Suppl 1):136.

64. Gumurdulu Y, Serin E, Ozer B, et al. Low eradication rate of Helicobacter pylori with triple 7-14 days and quadriple therapy in Turkey. World J Gastroenterol. 2004;10(5):668-671.

65. Perez Aldana L, Kato M, Nakagawa S, et al. The relationship between consumption of antimicrobial agents and the prevalence of primary Helicobacter pylori resistance. Helicobacter. 2002;7(5):306-309.

66. Vakil N. H. pylori treatment: new wine in old bottles? Am J Gastroenterol. 2009;104(1):26-30.

67. Megraud F. H. pylori antibiotic resistance: prevalence, importance, and advances in testing. Gut. 2004;53(9):1374-1384.

68. Megraud F. Helicobacter pylori and antibiotic resistance. Gut. 2007; 56(11):1502.

69. Fischbach L, Evans EL. Meta-analysis: the effect of antibiotic resistance status on the efficacy of triple and quadruple first-line therapies for Helicobacter pylori. Aliment Pharmacol Ther. 2007;26(3):343-357.

70. Gisbert JP, Pajares JM. Review article: Helicobacter pylori "rescue" regimen when proton pump inhibitor-based triple therapies fail. Aliment Pharmacol Ther. 2002;16(6):1047-1057.

71. Gisbert JP, Pajares JM. Helicobacter pylori "rescue" therapy after failure of two eradication treatments. Helicobacter. 2005;10(5):363-372.

72. Vakil N, Vaira D. Sequential therapy for Helicobacter pylori: time to consider making the switch? JAMA. 2008;300(11):1346-1347.

73. Houben MH, van de Beek D, Hensen EF, Craen AJ, Rauws EA, Tytgat GN. A systematic review of Helicobacter pylori eradication therapy - the impact of antimicrobial resistance on eradication rates. Aliment Pharmacol Ther. 1999;13(8):1047-1055.

74. Zullo A, Vaira D, Vakil N, et al. High eradication rates of Helicobacter pylori with a new sequential treatment. Aliment Pharmacol Ther. 2003; 17(5):719-726.

75. Molina-Infante J, Pazos-Pacheco C, Vinagre-Rodriguez G, et al. Non-bismuth quadruple "concomitant" therapy versus standard triple therapy for clarithromycin-susceptible $\mathrm{H}$. pylori and versus quadruple "sequential" therapy for clarithromycin-resistant H. pylori. Helicobacter. 2011;16(Suppl 1):135.

76. O'Morain CA, O'Connor JP. Is sequential therapy superior to standard triple therapy for the treatment of Helicobacter pylori infection? Nat Clin Pract Gastroenterol Hepatol. 2009;6(1):8-9.
77. Essa AS, Kramer JR, Graham DY, Treiber G. Meta-analysis: four-drug, three-antibiotic, non-bismuth-containing "concomitant therapy" versus triple therapy for Helicobacter pylori eradication. Helicobacter. 2009;14(2):109-118.

78. Calvet X, Gene E, Lopez T, Gisbert JP. What is the optimal length of proton pump inhibitor-based triple therapies for $\mathrm{H}$. pylori? A cost-effectiveness analysis. Aliment Pharmacol Ther. 2001;15(7): 1067-1076.

79. de Boer WA, Kuipers EJ, Kusters JG. Sequential therapy; a new treatment for Helicobacter pylori infection. But is it ready for general use? Dig Liver Dis. 2004;36(5):311-314.

80. Liou JM, Lin JT, Chang CY, et al. Levofloxacin-based and clarithromycin-based triple therapies as first-line and second-line treatments for Helicobacter pylori infection: a randomised comparative trial with crossover design. Gut. 2010;59(5):572-578.

81. Peitz U, Sulliga M, Wolle K, et al. High rate of post-therapeutic resistance after failure of macrolide-nitroimidazole triple therapy to cure Helicobacter pylori infection: impact of two second-line therapies in a randomized study. Aliment Pharmacol Ther. 2002;16(2):315-324.

82. Beales IL. Efficacy of Helicobacter pylori eradication therapies: a single centre observational study. BMC Gastroenterol. 2001;1:7.

83. Gisbert JP, Morena F. Systematic review and meta-analysis: levofloxacin-based rescue regimens after Helicobacter pylori treatment failure. Aliment Pharmacol Ther. 2006;23(1):35-44.

84. Gisbert JP, Bermejo F, Castro-Fernandez M, et al. Second-line rescue therapy with levofloxacin after $\mathrm{H}$. pylori treatment failure: a Spanish multicenter study of 300 patients. Am J Gastroenterol. 2008;103(1):71-76.

85. Gisbert JP, Castro-Fernandez M, Bermejo F, et al. Third-line rescue therapy with levofloxacin after two H. pylori treatment failures. Am J Gastroenterol. 2006;101(2):243-247.

86. Zullo A, De Francesco V, Hassan C. Second-line treatment for Helicobacter pylori eradication after sequential therapy failure: a pilot study. Therapy. 2006;3:251-254.

87. Perna F, Zullo A, Ricci C, Hassan C, Morini S, Vaira D. Levofloxacinbased triple therapy for Helicobacter pylori re-treatment: role of bacterial resistance. Dig Liver Dis. 2007;39(11):1001-1005.

88. Gisbert JP, Vinagre-Rodriguez G, Marin AC, Molina-Infante J. Second-line rescue triple therapy with levofloxacin after quadruple nonbismuth "sequential" or "concomitant" treatment failure. Helicobacter. 2011;16(Suppl 1):135.

89. Zullo A, Hassan C, Morini S, et al. Sequential therapy for H. pylori: an 'aberrant' therapy ready for general use. Dig Liver Dis. 2004;36(12):852-853; author reply 853.

90. Scaccianoce G, Hassan C, Panarese A, Piglionica D, Morini S, Zullo A. Helicobacter pylori eradication with either 7-day or 10-day triple therapies, and with a 10-day sequential regimen. Can J Gastroenterol. 2006;20(2):113-117.

91. Shehada S, Srugo I, Shaoul R. Failure of sequential therapy to eradicate Helicobacter pylori in previously treated subjects. Helicobacter. 2007;12(6):645-647.

92. Gisbert JP. Rescue therapy after Helicobacter pylori eradication failure. Gastroenterol Hepatol. 2011;34(2):89-99.
Clinical and Experimental Gastroenterology

\section{Publish your work in this journal}

Clinical and Experimental Gastroenterology is an international, peerreviewed, open access journal, publishing all aspects of gastroenterology in the clinic and laboratory, including: Pathology, pathophysiology of gastrointestinal disease; Investigation and treatment of gastointestinal disease; Pharmacology of drugs used in the alimentary tract;

\section{Dovepress}

Immunology/genetics/genomics related to gastrointestinal disease. This journal is indexed on CAS. The manuscript management system is completely online and includes a very quick and fair peer-review system. Visit http://www.dovepress.com/testimonials.php to read real quotes from published authors. 